\title{
WHERE TO FIND THE IMAGE OF A DERIVATION
}

\author{
MARTIN MATHIEU \\ Mathematisches Institut der Universität Tübingen \\ Auf der Morgenstelle 10, D-72076 Tübingen, Germany
}

\begin{abstract}
With this paper, we intend to provide an overview of some recent work on a problem on unbounded derivations of Banach algebras that still defies solution, the noncommutative Singer-Wermer conjecture. In particular, we discuss several global as well as local properties of derivations entailing quasinilpotency in the image.
\end{abstract}

1. Where to look for ... Derivations may serve as the generators of reversible evolutions of a physical system, say, if this is modelled by a Banach algebra. Not only historically, this point of view gave a strong impetus to the investigation of derivations and of how their properties relate to the structure of Banach algebras. The easiest examples one encounters are the inner derivations $\delta_{a}: x \mapsto x a-a x$, where $a$ is a given element in the Banach algebra $A$, and one may be tempted to think that there are no others. Indeed, one is morally right: a non-vanishing first cohomology group is generally considered rather as an obstacle than a delight, as well as philosophically: often derivations become inner in a larger Banach algebra. Here is an example. Let us suppose that $A$ is unital. (Since every derivation $\delta$ vanishes on the identity, this is no restriction of generality and will therefore be tacitly assumed henceforth.) Identifying $A$ with a closed subalgebra of the algebra $B$ of all bounded linear operators on $A$ via the left regular representation $a \mapsto L_{a}$, one has

$$
\left[L_{a}, \delta\right](x)=a \delta(x)-\delta(a x)=-\delta(a) x=L_{-\delta(a)}(x) \quad(x \in A),
$$

that is, $\left[L_{a}, \delta\right]=L_{-\delta(a)}$ or, under the above identification, $\delta(a)=\delta_{-\delta}(a)$ for all $a \in A$. Of course, to this end we have to assume that $\delta$ is bounded. This already indicates that the actual problem is with unbounded derivations, but as is well known, even bounded derivations in general need not be inner in the strict sense

1991 Mathematics Subject Classification: Primary 47B47; Secondary 46-02, 46H99, 47-02.

The paper is in final form and no version of it will be published elsewhere. 
even on very nice Banach algebras such as the compact operators on a separable Hilbert space.

Every inner derivation is a commutator: $\delta_{a}=[\cdot, a]$, and this notion is obviously vacuous in the commutative case. Still believing that derivations ought to be inner, they therefore should be rare on commutative Banach algebras. There was some evidence for this idea in the late 1940's and early 50's. Singer proved that there are no non-zero (bounded or unbounded) derivations on commutative $C^{*}$-algebras, and Singer and Wermer obtained the result that every bounded derivation on a commutative Banach algebra has its image in the radical [37]. Hence, the hunch was right up to semisimplicity. They also stated what soon was called the Singer-Wermer conjecture: The image of every derivation on a commutative Banach algebra lies in the radical. But this conjecture stood open for more than thirty years [39]. Out of it and other questions as well grew a new branch of functional analysis - automatic continuity theory, which helped to affirm the conjecture in the semisimple case, for example; [11], [18]. Nowadays, Thomas' theorem yields the full picture: Every derivation on a commutative Banach algebra is inner modulo the radical $\operatorname{rad}(A)$. Hence, derivations on Banach algebras (if everywhere defined) genuinely belong to the non-commutative setting.

There are also purely algebraic pieces of evidence for the assertion that derivations try to avoid commutativity, some of which only recently came to the attention of analysts. The most basic of these is the so-called Posner's second theorem [28, Theorem 2]: If $R$ is a prime ring and $\delta: R \rightarrow R$ is a non-zero derivation with the property that $\delta(a)$ commutes with $a$ for each $a \in R$, then $R$ has to be commutative. This was used in [25] to obtain the following result in the non-commutative setting. Call a derivation satisfying the above property commuting. Then every bounded commuting derivation on a Banach algebra maps into the radical. In fact, invoking Thomas' theorem, the boundedness assumption can be dropped as was shown in [26]. After these results were accomplished a number of mathematicians tried to affirm the non-commutative Singer-Wermer conjecture which we will state in the following way.

CONJECTURE 1. Every derivation on a Banach algebra which is commuting modulo the radical is inner modulo the radical.

This formulation is readily seen to be equivalent to the following one: if $\delta$ has the property that $[a, \delta(a)] \in \operatorname{rad}(A)$ for all $a \in A$, then $\delta A \subseteq \operatorname{rad}(A)$. For, if such a derivation equals the inner derivation $\delta_{a} \operatorname{modulo} \operatorname{rad}(A)$, then $\delta-\delta_{a} \operatorname{maps} A$ into $\operatorname{rad}(A)$ whence $\delta=\delta-\delta_{a}+\delta_{a}$ shows that $\delta$ leaves $\operatorname{rad}(A)$ invariant. By Johnson's and Sinclair's theorem [19], the induced derivation on the semisimple Banach algebra $A / \operatorname{rad}(A)$ is bounded, and being commuting, vanishes by [25, Theorem 3.2]. Thus, $\delta A \subseteq \operatorname{rad}(A)$.

So far, to our knowledge, Conjecture 1 has resisted all efforts to prove it. This will not be surprising if one knows that it is closely related to several other open questions having tantalised the Banach algebraists for some time. In his 
paper [10], Cusack compiled the following properties derivations on a Banach algebra may enjoy:

(1) Every derivation on a Banach algebra leaves each primitive ideal invariant,

(2) Every derivation on a Banach algebra has a nilpotent separating space,

(3) Every derivation on a semiprime Banach algebra is continuous,

to which we add

(4) Every derivation on a prime Banach algebra is continuous.

Cusack showed that if any of these properties fails, then there exists a topologically simple radical Banach algebra, no example of which is known and, in fact, is not expected to exist. By [26, Theorem 2], (2), (3), and (4) are equivalent (see also [14]), and the reduction procedure used in [26] shows that if (4) holds, then Conjecture 1 is valid; compare [33, Corollary 1.9] as well as [31] for the commutative case. In the same vein, (2) implies (1) [10, Theorem 4.5] which in turn entails Conjecture 1 by Posner's theorem. Unpublished work by Thomas (compare [32, Theorem 4.2.3] and [33, Theorem 1.8]) enables us to add a further property which is equivalent to (1):

(5) Every derivation on the unitisation of a radical Banach algebra leaves the radical invariant.

Therefore, if Conjecture 1 is true, then (1) holds. For, $[a+\lambda 1, \delta(a+\lambda 1)]=$ $[a, \delta(a)] \in \operatorname{rad}(A)$ for every derivation $\delta$ on the unitisation $A$ of a radical Banach algebra $A_{0}=\operatorname{rad}(A)$ and $a \in A_{0}$, whence by Conjecture $1, \delta A \subseteq \operatorname{rad}(A)$. This shows that our formulation of the non-commutative Singer-Wermer conjecture is equivalent to the one given in [33] and [40].

What is known about Conjecture 1 in its various formulations? Sinclair proved in [36, Theorem 2.2] that (1) holds for bounded derivations, and therefore Conjecture 1 holds for all derivations on semisimple Banach algebras (which, of course, is a special case of [26, Theorem 1]). Hence, the bounded case fits exactly into our philosophy. Partial results of [26, Theorem 1] had been obtained earlier by Yood [49, Theorem 3.1], Brešar and Vukman [7, Theorem 2.2], and Brešar [4, Corollary 3.7]. The way out of the dilemma arising when one wants to reduce Conjecture 1 to the semisimple case (and thus already needs to know that (1) holds) is provided by appealing to prime instead of primitive ideals. It is well-known (to algebraists, see [12, 3.3.2], [15, Proposition 1.1] or [9], and was reproved in [26, Lemma]) that minimal prime ideals are invariant under derivations of Banach algebras, which, together with automatic continuity results (e.g. [10, Lemma 2.3]) and Posner's and Thomas' theorems, allows one - in principle - to reduce to the case of a prime Banach algebra. This end, however, requires a slightly stronger assumption than in Conjecture 1, viz. that $\delta$ is commuting modulo the nilradical $\operatorname{nil}(A)$ of $A$ (i.e., the intersection of all prime ideals of $A$ ). This result seems, at present, to be the strongest evidence for the validity of Conjecture 1, and was 
obtained independently in [23, Theorem 2.2] and [32, Theorems 4.2.7 and 4.2.8].

THEOREM 1. Conjecture 1 holds for derivations commuting modulo the nilradical.

Since $\operatorname{nil}(A)$ is invariant under every derivation (which follows from the invariance of minimal prime ideals and can also be proved in other ways, see e.g. [10, Lemma 4.1]) it is close at hand to reduce to the semiprime situation; however, $\operatorname{nil}(A)$ need not be closed and the separating space may not be nilpotent, cf. (2) above. If $A$ is a semiprime Banach algebra, then the hypothesis in Conjecture 1 can be (formally) weakened. A derivation $\delta$ is said to be centralising if $[a, \delta(a)] \in Z(A)$, the centre of $A$, for all $a \in A$. It is not difficult to see that each centralising derivation is commuting modulo the nilradical (this, in fact, holds for every ring [23, Proposition 1.1], but in a Banach algebra already $\left.[a, \delta(a)]^{2}=0\right)$. In particular, each centralising derivation on a semiprime Banach algebra is commuting. This can be strengthened using [5, Corollary 1$]$ which implies that every additive mapping $F$ on a prime Banach algebra $A$ such that $[a,[a, F(a)]] \in Z(A)$ for all $a \in A$ is already commuting. Hence, each derivation $\delta$ on $A$ such that $[a,[a, \delta(a)]]$ is central modulo nil $(A)$ is actually commuting modulo nil $(A)$. This explains that the weaker assumption $[a,[a, \delta(a)]] \in \operatorname{nil}(A)$ implies that $\delta A \subseteq \operatorname{rad}(A)$ in $[32$, Theorem 4.2.7].

The same reasoning applies whenever $J$ is a $\delta$-invariant ideal which is contained in $\operatorname{rad}(A)$; one may without loss of generality assume that $J$ is semiprime since its nilradical nil $(J)$ (the intersection of all prime ideals containing $J$ ) will be $\delta$-invariant and contained in $\operatorname{rad}(A)$, if $J$ is. That is, $[a,[a, \delta(a)]] \in Z(A)(\bmod J)$ implies that $\delta A \subseteq \operatorname{rad}(A)$; compare [23, Concluding Remark] as well as [32, Theorem 4.2.8]. However, all these weakenings of the hypothesis in Conjecture 1 are merely of a formal nature since $\delta A \subseteq \operatorname{rad}(A)$ yields that $[a, \delta(a)] \in \operatorname{rad}(A)$ for all $a \in A$, and the challenging open question is whether the converse holds.

The results described above use the $\delta$-invariance of minimal prime ideals to reduce the problem either to the case of a bounded derivation on an arbitrary Banach algebra or to an arbitrary derivation on a prime Banach algebra, in which case the hypothesis creates the following dichotomy: either $\delta=0$, or the algebra is commutative (so that Thomas' theorem applies). The latter step is formalised in [32, Lemmas 4.2.5 and 4.2.6] (see also [33]) to the framework of differential polynomial identities $p\left(a, \delta(a), \delta^{2}(a), \ldots, \delta^{n}(a)\right)=0$ for all $a \in A$, where $p$ is a complex polynomial in $n+1$ non-commuting variables. The algebraic passepartout replacing Posner's theorem then becomes

(6) $p\left(a, \delta(a), \ldots, \delta^{n}(a)\right)=0$ and $A$ prime $\Rightarrow \delta=0$ or $A$ commutative,

whence the assumption in Theorem 1 may be replaced by $p\left(a, \delta(a), \ldots, \delta^{n}(a)\right) \in$ $\operatorname{nil}(A)$ for all $a \in A$. We list a number of examples of (6) recently studied:

$$
\begin{aligned}
& p\left(x_{1}, x_{2}\right)=\left[x_{1}, x_{2}\right]^{2} \text { and } p\left(x_{1}, x_{2}, x_{3}\right)=\left[x_{1}, x_{2}+x_{3}\right][8] \\
& p\left(x_{1}, x_{2}\right)=x_{2}^{2}[25], \text { as well as }
\end{aligned}
$$




$$
\begin{aligned}
& p\left(x_{1}, x_{2}\right)=\left[x_{1},\left[x_{1},\left[x_{1}, x_{2}\right]\right]\right][33],[44] \\
& p\left(x_{1}, x_{2}\right)=\left[x_{1},\left[x_{1}, x_{2}\right]\right][43] \text { and } p\left(x_{1}, x_{2}\right)=\left[x_{1}, x_{2}\right] x_{2}[45] .
\end{aligned}
$$

All these are refinements of $p\left(x_{1}, x_{2}\right)=\left[x_{1}, x_{2}\right]$, the case investigated by Posner [28], and all are restraints on the image of $\delta$ by comparing with another derivation. In fact, Posner's first theorem [28, Theorem 1] states that the image of a non-zero derivation $\delta$ on a prime algebra $A$ is "large" in the sense that no other non-zero derivation $\delta^{\prime}$ can vanish on $\delta A\left({ }^{1}\right)$, while his second theorem is an immediate consequence [24]. In the case of a general Banach algebra $A$, this behaviour remains valid modulo semiprime ideals. The arguments used to prove [23, Proposition 1.2 and Corollary 1.3] yield the following.

Proposition 2. Let $J$ be a semiprime ideal in $A$. The following conditions on a derivation $\delta$ on $A$ are equivalent.

(a) $[a, \delta(a)] \in J$ for all $a \in A$;

(b) $[b, \delta(a)] \in J$ for all $a, b \in A$;

(c) $\delta_{b}(c) A \delta(a) \subseteq J$ for all $a, b, c \in A$.

Calling a derivation $\delta$ satisfying (a) commuting modulo $J$, we realise that the image of every such derivation is mapped into $J$ by all inner derivations (condition (b)). Moreover, if $\delta$ is inner modulo $J$, i.e. $\left(\delta-\delta_{b}\right) A \subseteq J$ for some $b \in A$, then condition (c) becomes

$$
\delta(c) A \delta(a) \subseteq J \quad(a, c \in A)
$$

whence $\delta A \subseteq J$ by the semiprimeness of $J$. This, in particular, shows directly that " $\delta$ inner modulo $\operatorname{rad}(A)$ " is equivalent to " $\delta A \subseteq \operatorname{rad}(A)$ " (see above). It also leads to the question of locating the image of a derivation using other (semiprime) ideals than $\operatorname{rad}(A)$.

Question. For which semiprime ideals $J$ of a Banach algebra $A$ does the following variant of Conjecture 1 hold: Every derivation which is commuting modulo $J$ is inner modulo $J$ ?

If we take a primitive ideal $P$ in Proposition 2 and assume that $[a, \delta(a)] \in P$ for all $a \in A$, then $\delta P \nsubseteq P$ will imply that $\delta_{b}(c) \in P$ for all $b, c \in A$ by condition (c) (as $P$ is a prime ideal). Consequently, $A / P$ is commutative and primitive, i.e., $A / P \cong \mathbb{C}$ so that $P$ has to be a maximal ideal. This may support the feeling that there can at most exist a commutative obstruction to the non-commutative Singer-Wermer conjecture (cf. also [23]).

2. ... and where to find. Once we aim to learn about the image, i.e., the global behaviour of a derivation $\delta$, we need to understand local properties of $\delta$, i.e., those of $\delta(a)$ for some $a \in A$. Again, we start with a historical remark. In the process of developing a mathematical description of quantum mechanics the question came up whether bounded observables can fulfil the Heisenberg uncertainty

$\left(^{1}\right)$ This was observed in other contexts independently, see e.g. [13] and [47]. 
principle. The negative answer given in the framework of Hilbert space operators by Wintner in 1947 [48] was extended to the setting of the Banach algebra $L(E)$ of all bounded linear operators on a Banach space $E$ by Wielandt [46] and can be phrased as follows: No derivation on $L(E)$ attains a non-zero central value. In order to put this properly into its historical frame, we point out that every derivation $\delta$ on $L(E)$ is bounded (by semisimplicity) and inner, hence $\delta=\delta_{b}$ for some $b \in L(E)$, and also that $Z(L(E))=\mathbb{C} 1$. Therefore, Wielandt's result is an immediate consequence of the Kleinecke-Shirokov theorem:

THEOREM 3 [21], [35]. Every commutator $[a, b]$ in a Banach algebra satisfying $[a,[a, b]]=0$ is quasinilpotent.

Consequently, $\delta_{b}(a) \in Z(L(E))$ implies that $\delta_{b}(a)$ is both scalar and quasinilpotent, thus $\delta_{b}(a)=0$. Theorem 3 had been conjectured by Kaplansky in the early 50's on the base of the Wielandt-Wintner theorem and an older result due to Jacobson [17] stating that every commutator $[a, b]$ in an algebraic algebra over a field of characteristic zero satisfying $[a,[a, b]]=0$ is nilpotent (that is, Theorem 3 in the finite-dimensional case). A number of mathematicians struggled with Kaplansky's conjecture - we mention here only the partial results obtained by Halmos [16], Putnam [30], and Vidav [42] — until Kleinecke and Shirokov independently corroborated it. It is readily seen (cf. [20, p.20] or [25, Theorem 2.2]) that the inner derivation $\delta_{b}$ in Theorem 3 can be replaced by an arbitrary bounded derivation. As a result, we note the following property.

Proposition 4. No derivation on a primitive Banach algebra attains a nonzero central value.

The variant of Theorem 3 for bounded derivations immediately implies the Singer-Wermer theorem, which itself had been another conjecture by Kaplansky based on Shilov's result from 1947 stating that $C^{\infty}[0,1]$ cannot be normed as a Banach algebra [34]. Likewise, Proposition 4 together with the $\delta$-invariance of primitive ideals (condition (1) above) would imply Conjecture 1 without more ado.

It appears pertinent to state the following unsolved problem.

Conjecture 2. Let $\delta$ be a derivation on a Banach algebra $A$. For each $a \in A$, $[a, \delta(a)]=0$ implies that $\delta(a)$ is quasinilpotent.

This unbounded Kleinecke-Shirokov theorem (first conjectured in [22]) holds whenever Conjecture 1 is true. For, if $\delta P \subseteq P$ for every primitive ideal $P$ of $A$, then $[a, \delta(a)]=0$ implies that $\left[a+P, \delta_{P}(a+P)\right]=0\left(\right.$ where $\delta_{P}(x+P)=\delta(x)+P$ denotes the induced derivation on $A / P)$, and $\delta_{P}$ being bounded then yields that $\delta_{P}(a+P)$ is quasinilpotent. Now,

$$
\sigma_{A}(x)=\bigcup_{P} \sigma_{A / P}(x+P),
$$


where $\sigma_{B}(\cdot)$ denotes the spectrum with respect to the unital algebra $B$ and the union is taken over all primitive ideals of $A$, shows that $\delta(a)$ is quasinilpotent, too.

On the other hand, the validity of Conjecture 2 would imply Thomas' theorem, i.e., the commutative Singer-Wermer conjecture immediately and by means of this provide an alternative proof of Theorem 1 .

What is known about Conjecture 2? By [40, Proposition 1.10], the set of primitive ideals which are not invariant under a given derivation $\delta$ is finite and each of these exceptional primitive ideals is of finite codimension. Thus, by (7), $\sigma_{A}(\delta(a))$ is finite for each $a \in A$ such that $[a, \delta(a)]=0$. This is also observed in [33, Lemma 3.1]. In the same paper, Runde proves that if Conjecture 2 fails, then there is a quasinilpotent element $a$ such that $[a, \delta(a)]=0$ and $\delta(a)$ is invertible. He also affirms Conjecture 2 under various stronger hypotheses, e.g. [33, Theorem 3.5] reads as follows.

THEOREM 5. Conjecture 2 holds for all $a \in A$ such that $\left[a, \delta^{n}(a)\right]=0$ for all $n \in \mathbb{N}$.

Let us have a look at a proof of Conjecture 2 in the bounded case. Using the reduction to inner derivations as in the first paragraph of Section 1, it suffices to prove Theorem 3. Now, the assumption $[a,[a, b]]=0$ can also be written as $\delta_{a}^{2}(b)=0$ and the conclusion as $\delta_{a}(b)$ is quasinilpotent. In fact, this remains true for arbitrary bounded derivations in place of $\delta_{a}$ (see [25, Theorem 2.1] or [27, Theorem 2.1]). As a result, by (7), $\sigma_{A}(\delta(a))$ is finite for all $a \in A$ such that $\delta^{2}(a)=0$ whenever $\delta$ is any derivation on $A$. This was considerably strengthened in $[40$, Theorem 2.9]:

TheOrem 6. For all $a \in A, \delta^{2}(a)=0$ implies that $\delta(a)$ is quasinilpotent.

Theorem 6 implies Conjecture 1 in the commutative case (that is, Thomas' theorem) and appears to give some more evidence for the validity of Conjecture 2 . Note that the "globalisation" of Theorem 6 is easy to obtain: whenever $\delta^{2} A \subseteq$ $\operatorname{rad}(A)$, then $\delta A \subseteq \operatorname{rad}(A)$. This merely uses the semiprimeness of $\operatorname{rad}(A)$ (and hence is true for an arbitrary semiprime ideal $J$ of $A$ ) as well as the identity

$$
2 \delta(a) b \delta(a)=\delta^{2}(a b a)-a \delta^{2}(b a)-\delta^{2}(a b) a+a \delta^{2}(b) a \quad(a, b \in A) .
$$

From this and Thomas' theorem, we can deduce the following extension of Proposition 4.

THEOREM 7. Every derivation which maps into the centre maps into the radical.

For, if $\delta A \subseteq Z(A)$, then $\delta_{\mid Z(A)}$ maps $Z(A)$ into $\operatorname{rad}(Z(A))=Z(A) \cap \operatorname{rad}(A)$ and therefore $\delta^{2} A \subseteq \operatorname{rad}(A)$. By the above, $\delta A \subseteq \operatorname{rad}(A)$ follows. This observation is the global generalisation of Wielandt's result to arbitrary Banach algebras.

In each case, the local information one obtains is that $\delta(a) \in Q(A)$, the set of quasinilpotent elements, for certain $a \in A$. If, however, $\delta(a) \in Q(A)$ for all $a \in A$, then $\delta A \subseteq \operatorname{rad}(A)$. This result is due to Turovskiu and Shul'man [41] and 
was observed earlier by Pták [29] for inner derivations. There is an independent proof for the case of bounded derivations in [25] from which the general case can be derived as was pointed out to us by Runde (private communication). In fact, if $\delta A \subseteq Q(A)$ for an arbitrary derivation $\delta$ on $A$, then $\delta P \subseteq Q(A)$ for each primitive ideal $P$ of $A$. Thus, $(\delta P+P) / P$ is an ideal contained in $Q(A / P)$, and since $\operatorname{rad}(A / P)=0$, it follows that $(\delta P+P) / P=0$, i.e., $\delta P \subseteq P$. The bounded derivation $\delta_{P}$ on $A / P$ maps into $Q(A / P)$, and hence into $\operatorname{rad}(A / P)$. As a result, $\delta_{P}=0$ for all primitive ideals, whence $\delta A \subseteq \operatorname{rad}(A)$. This sequence of arguments yields a somewhat simpler proof than in [41].

Summarising the picture we tried to draw, we can now tell a reason why the image of a derivation could lie in the radical. A derivation on a Banach algebra tries to miss central elements (Proposition 4), and if it cannot, it is inner modulo the radical (Theorem 7). The reason for this is the Kleinecke-Shirokov theorem (Theorem 3) stating - at least in the bounded case, and if Conjecture 2 is true, then also in general - that a derivation $\delta$ maps the set $\{a \in A \mid[a, \delta(a)]=0\}$ into $Q(A)$. If this set is large enough (if $A$ is commutative, this is Thomas' theorem, if $A$ is arbitrary, this is Theorem 1$)$, then $\delta A \subseteq \operatorname{rad}(A)$. It is close at hand to believe that the only obstruction can come from the radical itself (Conjecture 1), and that therefore in this more general sense, derivations come as inner derivations. Yet, this is based on the conviction that an everywhere defined derivation on a Banach algebra is not too discontinuous, that is, its separating space lies in the radical.

3. Spectrally bounded operators. The hypotheses in the results and conjectures presented in the previous two sections were all designed for the ultimate conclusion that the image of a derivation is contained in the radical. We observed that some of the arguments work for other ideals as well, hence the question arises what can be said about the general range inclusion problem $\delta A \subseteq J$, where $J$ is a prescribed (closed) ideal of $A$ ? Wondering what turns the Jacobson radical into a distinguished ideal in this question, one notes that it combines both algebraic information (in terms of the representation theory) and analytic information (in terms of the spectral radius, i.e., generalised nilpotency) about the underlying Banach algebra, thus being very close to the kelson of this algebraic-analytic structure. It therefore does not come as a surprise that the Singer-Wermer conjecture is intimately tied to other deep structural questions about Banach algebras (see Section 1 above).

But still — why should $\delta A$ be contained in the radical? The purpose of this last section is to shed additional light onto this question from another viewpoint, opening up an alternative approach in the quest for a proof of the noncommutative Singer-Wermer conjecture. Recall that it suffices to prove that each image $\delta(a)$ is quasinilpotent, in particular not invertible. The reason for this is to be the Kleinecke-Shirokov theorem, or Conjecture 2, eventually. Starting from Wielandt's theorem, $[a, b] \neq 1$, we stressed the point that a derivation avoids non-zero central values; but this is not the whole truth. 
THEOREM 8. No derivation on a unital Banach algebra attains an invertible central value.

Theorem 8 holds in the bounded case by the Kaplansky-Kleinecke-Shirokov theorem and thus for semisimple Banach algebras, while Thomas' theorem settles the commutative case. If $z=\delta(a) \in Z(A)$ is invertible for some $a \in A$, then $\delta^{\prime}=$ $L_{z^{-1}} \delta$ is a derivation satisfying $\delta^{\prime}(a)=1$. But by Theorem 6 , this is impossible and therefore Theorem 8 holds in general.

The following consequence of Theorem 8 is the actual local generalisation of Wielandt's theorem and, in the commutative case, was the key to Thomas' proof of the Singer-Wermer conjecture [39].

Corollary 9. No derivation on a unital Banach algebra attains the value 1.

We like to point out that Conjecture 1 (in the formulation (1)) together with Proposition 4 would yield a "natural" argument for Theorem 8. If $\delta A \subseteq Q(A)$, then the image of $\delta$ in fact keeps away from 1 , that is, the distance $\mathrm{d}(1, \delta A)$ is at least 1. To our knowledge, not much is known about the closure $\overline{\delta A}$ of the image of a derivation $\delta$ on a general Banach algebra; the special case $A=L(H)$, $H$ a Hilbert space for example is treated in [1], [2], [38]. On the other hand, there of course have been purely algebraic investigations on derivations attaining invertible values, e.g. [3].

Let us assume commutativity for a moment because this case is the most clear. For each $a \in A$ there then is a maximal ideal $I$ of $A$ such that $\delta(a) \in I$. The Singer-Wermer conjecture, however, states much more: $\delta(a)$ is contained in all maximal ideals. Thus, a derivation destroys invertibility in a very strong sense. We use this as a motivation for the following concept.

Definition. Let $A, B$ be Banach algebras. A linear mapping $T: A \rightarrow B$ is called spectrally bounded if there is $M>0$ such that $r(T(a)) \leq M r(a)$ for all $a \in A$. If $M=1$, we say that $T$ is a spectral contraction, and $T$ is a spectral isometry if $r(T(a))=r(a)$ for all $a \in A$. Finally, $T$ is spectrally infinitesimal if $r(T(a))=0$ for all $a \in A$. (Herein, of course, $r(x)$ denotes the spectral radius of the element $x$.)

As the spectral radius is an algebra norm only in the commutative and semisimple case, the philosophy behind the notion of a spectrally bounded operator is to try using a "non-commutative seminorm" to measure some kind of boundedness with respect to invertibility rather than to continuity. There does not seem to be a thorough study of this class of operators, so we start with a few basic remarks.

There are of course unbounded spectrally bounded operators. For instance, each isomorphism between unital algebras is a spectral isometry and more generally every unital homomorphism is spectrally contractive. Likewise the left regular representation is a spectral isometry from a unital Banach algebra $A$ into $L(A)$ (a fundamental fact), and so is the canonical epimorphism $\pi: A \rightarrow A / \operatorname{rad}(A)$ (another fundamental fact). Nagasawa's theorem states that each surjective spectral 
isometry between commutative semisimple unital Banach algebras is an isomorphism $\left({ }^{2}\right)$.

Thomas' theorem states that every derivation on a commutative Banach algebra is spectrally infinitesimal, while it is clear that each spectrally bounded operator on a commutative Banach algebra leaves the radical invariant.

Proposition 10. Each spectrally bounded derivation fixes the radical.

For, if $r(\delta(a)) \leq M r(a)$ for some $M>0$ and all $a \in A$, then

$$
r(x \delta(a))=r(\delta(x a)-\delta(x) a)=r(\delta(x a)) \leq M r(x a)=0
$$

whenever $a \in \operatorname{rad}(A)$ and $x \in A$, whence $\delta \operatorname{rad}(A) \subseteq \operatorname{rad}(A)$. Hence, such a derivation $\delta$ induces a bounded derivation $\delta^{\prime}$ on the semisimple Banach algebra $A / \operatorname{rad}(A)$, and

$$
r\left(\delta^{\prime}(\pi(a))\right)=r(\pi(\delta(a)))=r(\delta(a)) \leq M r(a)=\operatorname{Mr}(\pi(a)) \quad(a \in A)
$$

shows that $\delta^{\prime}$ is spectrally bounded with the same constant. Clearly, $\delta$ is commuting modulo the radical if and only if $\delta^{\prime}$ is commuting, and $\delta A \subseteq \operatorname{rad}(A)$ if and only if $\delta^{\prime}=0$. Thus, Conjecture 1 is equivalent to

CONJECTURE $1^{\prime}$. Every derivation commuting modulo the radical is spectrally bounded.

Challenge: Prove Conjecture $1^{\prime}$ in the commutative case not using Thomas' theorem. This would yield a new proof of the commutative Singer-Wermer conjecture. Moreover, one would arrive at the following commutativity-free characterisation:

$$
\delta A \subseteq \operatorname{rad}(A) \Leftrightarrow \delta \text { is spectrally bounded }
$$

provided that the following is true.

CONJECTURE 3. Each spectrally bounded derivation is spectrally infinitesimal.

This conjecture was recently affirmed for inner derivations in [6] building on work by Pták [29]. Note that by the above, it suffices to ascertain Conjecture 3 for bounded derivations.

We conclude by recapitulating what is known about the following three conditions on a derivation $\delta$ defined on a Banach algebra $A$.

(a) $[a, \delta(a)] \in \operatorname{rad}(A)$ for all $a \in A$;

(b) $\delta$ is spectrally bounded;

(c) $\delta A \subseteq \operatorname{rad}(A)$.

We have $(\mathrm{a})+(\mathrm{b}) \Rightarrow(\mathrm{c}) \Rightarrow(\mathrm{a})+(\mathrm{b})$, the latter implication being trivial. The implication $(\mathrm{a}) \Rightarrow(\mathrm{c})$ is Conjecture 1 while $(\mathrm{b}) \Rightarrow(\mathrm{c})$ is Conjecture 3 . None of these seem to imply the other, in fact $(\mathrm{a}) \Rightarrow(\mathrm{b})$, i.e. Conjecture $1^{\prime}$, is the same as Conjecture 1.

$\left({ }^{2}\right)$ It is an open problem whether every spectrum-preserving linear surjection on a semisimple Banach algebra is a Jordan isomorphism. Such a map is known to be continuous and injective. 
Acknowledgements. The author is grateful to the participants of the Special Semester on Banach Algebras, in particular Bernard Aupetit, for several discussions on the topic of this paper.

Added in proof. 1. Combining the techniques used in [26] with a recent paper by C. Lanski (Proc. Amer. Math. Soc. 118 (1993), 731-734) it follows that the hypothesis in Theorem 1 can be replaced by the assumption that some higher commutator $[\ldots[x,[x, \delta(x)]] \ldots]$ is contained in the nilradical for all $x$.

2. A recent preprint by R. E. Curto and the present author contains an alternative argument for the result in [6] (i.e., Conjecture 3 for inner derivations) not relying on the subharmonicity of the spectral radius.

\section{References}

[1] C. Apostol, Inner derivations with closed range, Rev. Roumaine Math. Pures Appl. 21 (1976), 242-265.

[2] C. A postol and J. G. S tampfli, On derivation ranges, Indiana Univ. Math. J. 25 (1976), 857-869.

[3] J. Bergen, I. N. Herstein and C. Lanski, Derivations with invertible values, Canad. J. Math. 35 (1983), 300-310.

[4] M. Brešar, Centralizing mappings on von Neumann algebras, Proc. Amer. Math. Soc. 111 (1991), 501-510.

[5] —, On a generalization of the notion of centralizing mappings, ibid. 114 (1992), 641-649.

[6] -, Derivations decreasing the spectral radius, Arch. Math. (Basel) 61 (1993), 160-162.

[7] M. Brešar and J. Vukman, On left derivations and related mappings, Proc. Amer. Math. Soc. 110 (1990), 7-16.

[8] -, -, Derivations on noncommutative Banach algebras, Arch. Math. (Basel) 59 (1992), $363-370$.

[9] C.-L. Chuang and T.-K. Lee, Invariance of minimal prime ideals under derivations, Proc. Amer. Math. Soc. 113 (1991), 613-616.

[10] J. Cusack, Automatic continuity and topologically simple radical Banach algebras, J. London Math. Soc. 16 (1977), 493-500.

[11] H. G. Dales, Automatic continuity: a survey, Bull. London Math. Soc. 10 (1978), 129-183.

[12] J. Dixmier, Algèbres envellopantes, Cahier Sci. 27, Gauthier-Villars, Paris, 1974.

[13] C. K. Fong and A. R. Sourour, On the operator identity $\sum A_{k} X B_{k} \equiv 0$, Canad. J. Math. 31 (1979), 845-857.

[14] R. V. Garimella, On nilpotency of the separating ideal of a derivation, Proc. Amer. Math. Soc. 117 (1993), 167-174.

[15] K. R. Goodearl and R. B. Warfield, Primitivity in differential operator rings, Math. Z. 180 (1982), 503-524.

[16] P. R. Halmos, Commutators of operators, II, Amer. J. Math. 76 (1954), 191-198

[17] N. Jacobson, Rational methods in the theory of Lie algebras, Ann. of Math. 36 (1935), $875-881$

[18] B. E. Johnson, Continuity of derivations on commutative Banach algebras, Amer. J. Math. 91 (1969), 1-10.

[19] B. E. Johnson and A. M. Sinclair, Continuity of derivations and a problem of Kaplansky, ibid. 90 (1968), 1067-1073.

[20] I. Kaplansky, Functional analysis, in: Some Aspects of Analysis and Probability, Surveys Appl. Math. 4, New York, 1958, 1-34.

[21] D. C. Kleinecke, On operator commutators, Proc. Amer. Math. Soc. 8 (1957), 535-536 
[22] M. Mathieu, Is there an unbounded Kleinecke-Shirokov theorem?, Sem.ber. Funkt.anal. 18, Tübingen, 1990, 137-143.

[23] - On the range of centralising derivations, preprint, 1991.

[24] - Posner's second theorem deduced from the first, Proc. Amer. Math. Soc. 114 (1992), 601-602.

[25] M. Mathieu and G. J. Murphy, Derivations mapping into the radical, Arch. Math. (Basel) 57 (1991), 469-474.

[26] M. Mathieu and V. Runde, Derivations mapping into the radical, II, Bull. London Math. Soc. 24 (1992), 485-487.

[27] G. J. Murphy, Aspects of the theory of derivations, this volume, 267-275.

[28] E. C. Posner, Derivations in prime rings, Proc. Amer. Math. Soc. 8 (1957), 1093-1100.

[29] V. Pták, Commutators in Banach algebras, Proc. Edinburgh Math. Soc. 22 (1979), 207211.

[30] C. R. Putnam, On the spectra of commutators, Proc. Amer. Math. Soc. 5 (1954), 929931.

[31] V. Runde, Automatic continuity of derivations and epimorphisms, Pacific J. Math. 147 (1991), 365-374.

[32] —, Problems in automatic continuity, Ph.D. Thesis, Univ. California, Berkeley, 1993.

[33] —, Range inclusion results for derivations on noncommutative Banach algebras, Studia Math. 105 (1993), 159-172.

[34] G. Shilov, On a property of rings of functions, Dokl. Akad. Nauk SSSR 58 (1947), 985988 (in Russian).

[35] F. V. Shirokov, Proof of a conjecture of Kaplansky, Uspekhi Mat. Nauk. 11 (1956), 167-168 (in Russian).

[36] A. M. Sinclair, Continuous derivations on Banach algebras, Proc. Amer. Math. Soc. 20 (1969), 166-170.

[37] I. M. Singer and J. Wermer, Derivations on commutative normed algebras, Math. Ann. 129 (1955), 260-264.

[38] J. G. Stampfli, On the range of a hyponormal derivation, Proc. Amer. Math. Soc. 52 (1975), 117-120.

[39] M. P. Thomas, The image of a derivation is contained in the radical, Ann. of Math. 128 (1988), 435-460.

[40] - Primitive ideals and derivations on non-commutative Banach algebras, Pacific J. Math. 159 (1993), 139-152.

[41] Yu. V. Turovskiǔ and V. S. Shul'man, Conditions for massiveness of the range of the derivation of a Banach algebra and associated differential operators, Math. Notes 42 (1987), 669-674.

[42] I. Vidav, Über eine Vermutung von Kaplansky, Math. Z. 62 (1955), 330.

[43] J. Vukman, Commuting and centralizing mappings in prime rings, Proc. Amer. Math. Soc. 109 (1990), 47-52.

[44] -, On derivations in prime rings and Banach algebras, ibid. 116 (1992), 877-884.

[45] —, A result concerning derivations in noncommutative Banach algebras, Glas. Mat. 26 (1991), 83-88

[46] H. Wielandt, Über die Unbeschränktheit der Operatoren der Quantenmechanik, Math. Ann. 121 (1949/50), 21

[47] J. P. Williams, On the range of a derivation, Pacific J. Math. 38 (1971), 273-279.

[48] A. Wintner, The unboundedness of quantum-mechanical matrices, Phys. Rev. 71 (1947), $738-739$.

[49] B. Yood, Continuous homomorphisms and derivations on Banach algebras, in: F. Greenleaf and D. Gulick (eds.), Banach Algebras and Several Complex Variables, Contemp. Math. 32, Amer. Math. Soc., Providence, R.I., 1984, 279-284. 
Editorial note: See also the paper of V. S. Shul'man in this volume. The editor has been informed by Matej Brešar that Conjecture 3 was recently verified. Concerning the problem mentioned in footnote $\left({ }^{2}\right)$, some progress has recently been made by B. Aupetit and H. du T. Mouton in their paper Spectrum-preserving linear mappings in Banach algebras, Studia Math. 109 (1994), 91-100; see also the papers of B. Aupetit and A. R. Sourour in this volume. 